\title{
Novel lead-anchor technique using an active fixation quadripolar left ventricular lead in cardiac resynchronization therapy
}

\author{
Yukihiro Inamura ${ }^{1}$, Osamu Inaba ${ }^{1}$, Akira Sato ${ }^{1}$, Junichi Nitta ${ }^{2}$, Masahiko Goya ${ }^{3}$, and \\ Tetsuo Sasano ${ }^{4}$ \\ ${ }^{1}$ Japanese Red Cross Saitama Hospital \\ ${ }^{2}$ Sakakibara Heart Institute \\ ${ }^{3}$ Tokyo Medical and Dental University Hospital \\ ${ }^{4}$ Tokyo Medical and Dental University
}

November 18, 2021

\begin{abstract}
In this report, we present a case of successful advancement of an LV lead into tortuous vessels. This was achieved by deep engagement of the coronary sinus with a cannulation catheter by applying the anchor technique using the Medtronic Attain Stability Quad lead.
\end{abstract}

Novel lead-anchor technique using an active fixation quadripolar left ventricular lead in cardiac resynchronization therapy

Yukihiro Inamura $^{\mathrm{a}}$, MD, PhD; Osamu Inaba ${ }^{\mathrm{a}}, \mathrm{MD}, \mathrm{PhD}$ (oinbcvm@tmd.ac.jp); Akira Sato ${ }^{\mathrm{a}}$, MD (chezasato@gmail.com); Junichi Nitta ${ }^{\mathrm{b}}, \mathrm{MD}, \mathrm{PhD}$ (jnitta-ind@umin.ac.jp); Masahiko Goya ${ }^{\mathrm{c}}, \mathrm{MD}, \mathrm{PhD}$ (cameister58.cvm@tmd.ac.jp); Tetsuo Sasanoc ${ }^{c}, \mathrm{MD}, \mathrm{PhD}$ (sasano.cvm@tmd.ac.jp)

${ }^{a}$ Department of Cardiology, Japanese Red Cross Saitama Hospital, Saitama, Japan

${ }^{b}$ Department of Cardiology, Sakakibara Heart Institute, Tokyo, Japan

${ }^{\mathrm{c}}$ Department of Cardiology, Tokyo Medical and Dental University, Tokyo, Japan.

Funding

This research did not receive any specific grant from funding agencies in the public, commercial, or not-forprofit sectors.

\section{Disclosures}

None.

Conflict of interest: All authors have no conflicts of interest to declare.

Ethics approval: Written informed consent was obtained from the patient for publication of this case report and accompanying images.

Corresponding Author :

Yukihiro Inamura

Japanese Red Cross Saitama Hospital,1-5 Shintoshin, Chuo-ku, Saitama 330-8553, Japan 
E-mail: janes.ina929@gmail.com

\section{Introduction}

Cardiac resynchronization therapy (CRT) is an important treatment for patients with heart failure. ${ }^{1,2}$ In clinical practice, it is difficult to advance a left ventricular (LV) lead through narrow or tortuous vessels in some cases. In this report, we present a case of successful advancement of an LV lead into tortuous vessels. This was achieved by deep engagement of the coronary sinus with a cannulation catheter by applying the anchor technique using Medtronic Attain Stability Quad lead. This useful technique for LV lead delivery has not been previously reported in the literature.

\section{Case Report}

\section{History/examination}

A 55-year-old man presented with heart failure due to dilated hypertrophic cardiomyopathy. His electrocardiogram revealed a right bundle branch block with a wide QRS complex (QRS length=208 ms) and first-degree atrioventricular block (PR length $=316 \mathrm{~ms}$ ). He had undergone catheter ablation four times for atrial fibrillation/tachycardia and had taken diuretics and beta-blockers for the same. Despite the rhythm control and adequate medication therapy, he still had dyspnea and refractory leg edema. To manage the patient's uncontrolled heart failure, we decided to implant a CRT pacemaker. Written informed consent was obtained from the patient before the CRT pacemaker implantation.

\section{Differential diagnosis, investigation, and treatment}

Angiography of the coronary sinus was used to determine whether the posterolateral vein was suitable to deploy the LV lead. We selected the active fixation LV pacing lead (Attain Stability Quad 4798, Medtronic, Dublin, Ireland) for implantation, which is a quadripolar LV lead with an active fixation helix assembly, designed to position the lead in the coronary sinus. ${ }^{3}$ A large-curve-type coronary sinus cannulation catheter (Attain Command + SureValve Integrated Valve, Medtronic, Dublin, Ireland) was used to deploy the lead using a standard over-the-wire technique, which involved advancing the pacing lead over the wire into the desired location with the distal electrodes positioned in the mid-LV segment. However, the lead did not advance smoothly as the target vessel was tortuous (Figure 1). Moreover, the pacing threshold was high, despite the deep insertion of the LV lead. To overcome this, we used an extra support guidewire (GRAND SLAM, Asahi Intecc, Aichi, Japan) and a 135-degree subselection catheter (Attain Select II + SureValve sub-selection catheter, Medtronic); however, we were unable to advance the LV lead further.

Subsequently, we attempted to engage the cannulation catheter deep within the coronary sinus as this could provide better support to pass the LV lead through the tortuous vessel. To achieve this, we applied the anchor technique, which is already reported as a technique for obtaining superior guiding catheter support during the advancement of a balloon catheter in coronary angioplasty. ${ }^{4}$ Once the lead was placed in the deepest position, it was rotated clockwise to achieve active temporary fixation of the lead. Next, we inserted and advanced the cannulation catheter while gently pulling the temporarily fixed LV lead. As the LV lead was fixed, it did not fall out of position despite being pulled, and we were able to advance the cannulation catheter deeper. As a result, superior cannulation catheter support was obtained without the LV lead falling out of position. Following this, the LV lead was rotated counterclockwise, and the fixation mechanism was released from the vein wall. Further, the lead was advanced to the middle lateral position, and refixation was performed.

\section{Outcome and follow-up}

Using this method, the LV lead was successfully and smoothly passed through the tortuous vessel. We called this method the "lead anchor technique" (Figure 2). By obtaining superior cannulation catheter support, we were able to implant the LV lead at a low pacing threshold and without a phrenic nerve stimulation site (Figure 3). After the CRT implantation, the pacing threshold was not increased, QRS complex improved to $170 \mathrm{~ms}$, and heart failure could be controlled. 


\section{Discussion}

Cardiac resynchronization therapy relieves symptoms and decreases mortality in patients with heart failure, depressed LV systolic function, and prolonged QRS duration. ${ }^{5,6}$ To obtain maximum benefit from the CRT, it is important to place the LV lead in a suitable region, particularly in lateral and non-apical positions with a low pacing capture threshold, and without phrenic nerve stimulation. However, lead revision is required due to lead stability issues, such as incorrect pacing location, lead dislodgement, increase in pacing capture threshold, or phrenic nerve stimulation in up to $7 \%$ of the CRT implantations. ${ }^{7}$ To overcome these, active fixation LV leads have been developed. ${ }^{8}$ The Medtronic Attain Stability Quad lead, which combines an active fixation mechanism with a quadripolar lead, allows for a targeted approach to LV pacing. ${ }^{3,9,10}$ Using an active fixation lead allows for better stability in the veins, which often have large diameters, compared with a passive fixation lead, the stability of which concerns operators. ${ }^{11}$

It is also important to develop a technique to advance an LV lead through target vessels and to overcome lead stability issues. In clinical practice, it is sometimes difficult to advance an LV lead through narrow and tortuous vessels. In these cases, we use a subselection catheter, extra support guidewire, and an LV lead with minimum French size lead body diameter; however, these devices are sometimes ineffective. In such difficult cases, percutaneous coronary intervention techniques can be useful. For instance, in percutaneous coronary intervention, deep engagement of the guiding catheter could provide good support. Fujita et al. reported the use of the anchor method, in which deep engagement of the guiding catheter was achieved by pulling an anchor balloon inflated in a non-target vessel. Using this method, superior guiding catheter support was maintained even after balloon deflation, ${ }^{4}$ and Kumagai et al. has reported the balloon anchor method for CRT implantation. ${ }^{12}$ We applied this technique in the LV lead implantation without using balloon catheter. Temporary fixing of the lead was performed anterior to the target position. Pulling the fixed lead made the cannulation catheter advance further. This method provided superior cannulation catheter support without the LV lead falling out. Adjusting the cannulation catheter position could help the lead to advance further. It should be noted that pulling the lead too hard could cause the fixation helix to stretch. To avoid this, it was important to pull the lead as gently as possible. Moreover, if advancing the cannulation catheter was found to be too difficult, it should not be forced.

To our knowledge, this is the first study that reports the application of the anchor technique for LV lead implantation using the Medtronic Attain Stability Quad lead. The 'lead anchor' technique may help in the management of difficult cases of LV lead implantation. This novel technique could help the operators to efficiently advance LV leads through tortuous vessels during the CRT procedure.

\section{Acknowledgement:}

We would like to thank Editage (www.editage.jp) for English language editing.

\section{Authorship List:}

Author Contributions:

Author 1: Yukihiro Inamura, the corresponding author, drafted this manuscript.

Author 2: Osamu Inaba, conceived and designed the manuscript for important intellectual content.

Author 3: Akira Sato, provided advice on manuscript design.

Author 4: Junichi Nitta, contributed to discussion of the manuscript and provided advice on manuscript design.

Author 5: Masahiko Goya, provided advice on manuscript design.

Author 6: Tetsuo Sasano, contributed to discussion of the manuscript, final approval of the manuscript submitted.

\section{References}


1. Abraham, W.T., Fisher, W.G., Smith, A.L., et al. 2002. Cardiac resynchronization in chronic heart failure. N Engl J Med 346(24):1845-1853.

2. Clealand, J.D., Daubert, J.C., Erdmann, E., et al. 2005. The effect of cardiac resynchronization on morbidity and mortality in heart failure. N Engl J Med 352(15):1539-1549.

3. Keilegavlen, H., Hovstad, T., Faerestrand, S. 2016. Active fixation of a thin transvenous left-ventricular lead by a side helix facilitates targeted and stable placement in cardiac resynchronization therapy. Europace 18(8):1235-1240.

4. Fujita, S., Tamai, H., Kyo, E., et al. 2003. New technique for superior guiding catheter support during advancement of a balloon in coronary angioplasty: The Anchor Technique. Catheter and Cariovasc interv 59(4):482-488.

5. Bristow, M.R., Saxon, L.A., Boehmer, J., et al. 2004. Cardiac-resynchronization therapy with or without an implantable defibrillator in advanced chronic heart failure. New Engl J Med 350(21):21402150.

6. Moss, A.J., Hall, W.J., Cannom, D.S., et al. 2009. Cardiac-resynchronization therapy for the prevention of heart-failure events. N Engl J Med 361:1329-1338.

7. Gras, D., Böcker, D., Lunati, M., et al. 2007. Implantation of cardiac resynchronization therapy systems in the CARE-HF trial: orocedural success rate and safety. Europace 9(7):516-522

8. Crossley, G.H., Exner, D., Mead, R.H., et al. 2010. Chronic performance of an active fixation coronary sinus lead. Heart Rhythm 7(4):472-478.

9. Chapman, M., Bates, M.G.D., Behr, J.M., et al. 2019. A novel quadripolar active fixation leftventricular pacing lead for cardiac resynchronization therapy: initial United Kingdom experience. JACC Clin Electrophysiol 5(9):1028-1035.

10. Jackson, K.P., Faerestrand, S., Philippon, F., et al. 2020. Performance of a novel active fixation quadripolar left ventricular lead for cardiac resynchronization therapy: Attain Stability Quad Clinical Study results. J Cardiovasc Electrophysiol 31(5):1147-1154.

11. Keilegavlen, H., Schuster, P., Hovstad, T., Faerestrand, S. 2020. Performance of an active fixation bipolar left ventricular lead vs passive fixation quadripolar leads in cardiac resynchronization therapy. J Arrhythm 37(1):212-218.

12. Kumagai, Y., Arimoto, T., Yamauchi, S., Kutsuzawa, D., Tsuchiya, H., Watanabe, M. 2018. Implantation of a cardiac resynchronization therapy device using the anchor balloon technique in a patient with a tortuous coronary sinus branch. Heart Rhythm Case Reports 4:339-342.

\section{Figure legends}

Figure 1. Contrast venography demonstrating a tortuous posterolateral coronary sinus vessel.

Figure 2 A) The Medtronic Attain Stability Quad lead. The 5.6-French non-electrically active side helix is positioned between the third and fourth electrodes. This picture is provided by Medtronic Japan. B) Once the LV lead is positioned as deep as possible, it is rotated clockwise and active temporary fixation is performed (arrow ). C) Pulling the active fixation lead (arrow) while advancing the cannulation catheter deeper (arrow ). Deep engagement of the cannulation catheter is obtained without the LV lead falling out. D) After deep engagement, the lead is rotated counterclockwise, fixation mechanism is released from the vein wall, LV lead is advanced to the desired position (arrow), and refixation is performed. Deep engagement of the cannulation catheter provides backup support and helps the LV lead to cross the lesion.

$\mathrm{LV}$, left ventricular

Figure 3 . A) During the "lead anchor technique" is performed. Deep engagement of the cannulation catheter is obtained. B) After the "lead anchor technique" is performed. The last helix position within the coronary sinus. The pacing threshold was $1.25 \mathrm{~V} / 0.4 \mathrm{~ms}$ at LV1 to LV2.

LV, left ventricular 
Figure 1

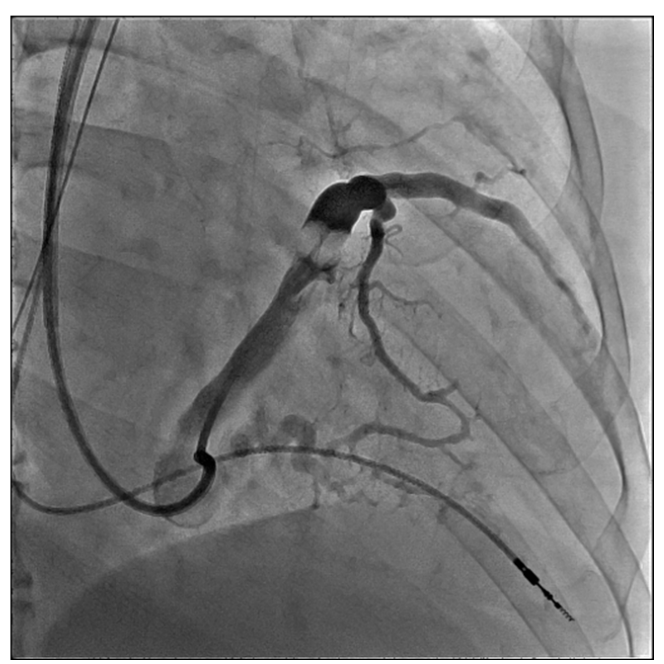

Figure 2
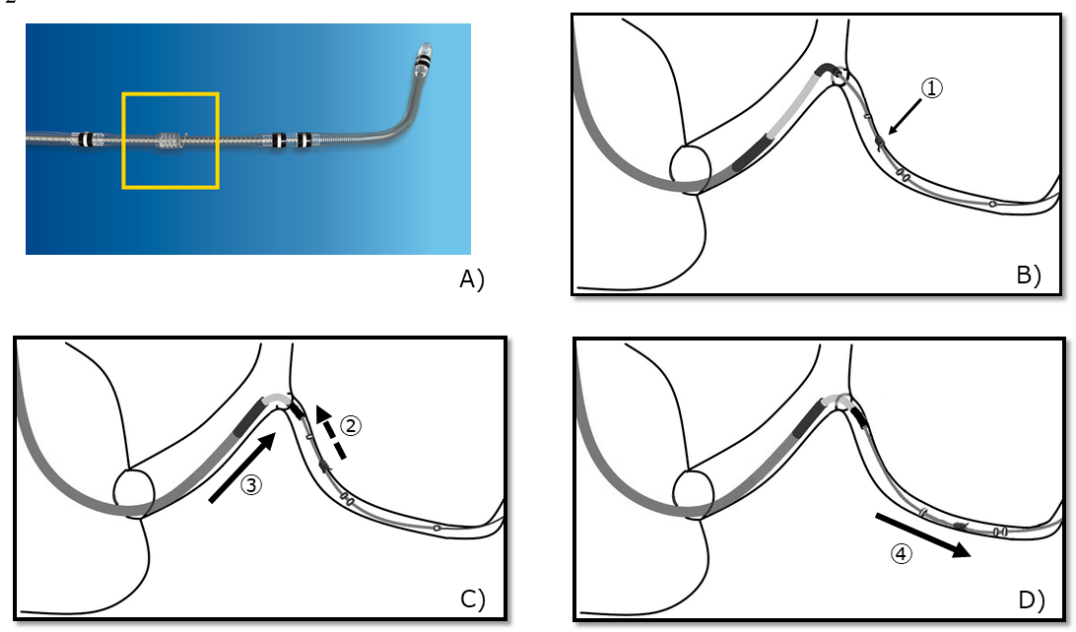

Figure 3
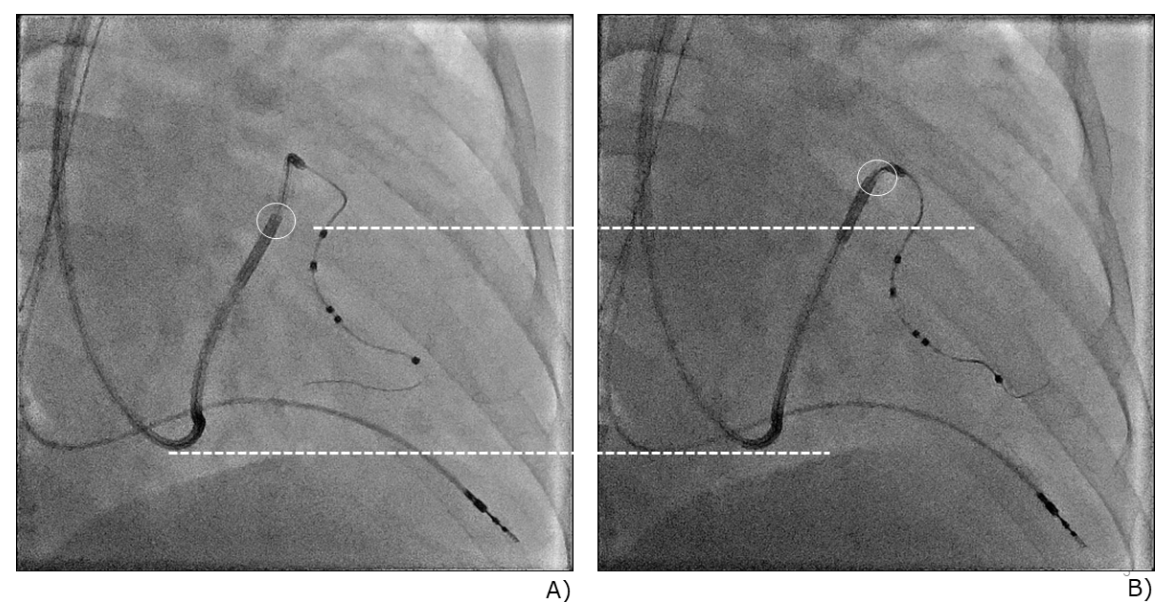